\title{
Mercaptopurine in childhood leukaemia: the effects of dose escalation on thioguanine nucleotide metabolites
}

\begin{abstract}
L. LENNARD ${ }^{1}$, J. WELCH ${ }^{2}$ \& J. S. LILLEYMAN
${ }^{1}$ University of Sheffield Department of Medicine and Pharmacology, Section of Molecular Pharmacology and Pharmacogenetics, The Royal Hallamshire Hospital, Sheffield S10 2JF, ${ }^{2}$ University of Sheffield, Department of Paediatrics, Section of Paediatric Haematology, The Children's Hospital Sheffield, S10 2TH and Department of Paediatric Oncology, St Bartholomew's and the Royal London Hospital School of Medicine, St Bartholomew's Hospital, London EC1A 7BE
\end{abstract}

The current U.K. trial protocol (UKALL XI) for childhood lymphoblastic leukaemia demands mercaptopurine (MP) dose escalation in children who tolerate daily $75 \mathrm{mg} / \mathrm{m}^{2}$ MP $(100 \%$ dose $)$ without cytopenias. The previous trial (UKALL X) did not. MP metabolism was studied in a group of UKALL XI children $(n=21)$ who tolerated $100 \%$ dosages and who were matched in this respect with a similar group of UKALL X children. Red blood cell MP derived thioguanine nucleotide (TGN) concentrations were measured in both groups under comparable conditions; at $75 \mathrm{mg} / \mathrm{m}^{2} \mathrm{MP}$ there was no significant difference. MP dose escalation in the UKALL XI children produced higher TGN concentrations (TGNs at $100 \%$ vs $125 \%$ dosages, median difference $90 \mathrm{pmol} / 8 \times 10^{8} \mathrm{RBCs}, 95 \%$ CI 25 to $\left.165 \mathrm{pmol}, P<0.02\right)$. Assayed at the time of cytopenia induced dose reduction, the UKALL XI children had accumulated significantly higher TGN concentrations than the UKALL $X$ children (median difference $78 \mathrm{pmol} / 8 \times 10^{8} \mathrm{RBCs}, 95 \%$ CI 20 to $144, P<0.02$ ). These findings indicate that dose escalation in children tolerant of $100 \%$ MP dosages produces higher peak TGN concentrations.

Keywords childhood leukaemia thioguanine nucleotides mercaptopurine compliance

\section{Introduction}

Mercaptopurine (MP) is universally used in the continuing chemotherapy of standard risk 'acute' lymphoblastic leukaemia (ALL) in children. The anti-leukaemic effect of MP can be related to drug derived thioguanine nucleotides (TGNs) [1]. Children vary in their ability to produce TGNs, and those who fail to form adequate amounts are at an increased risk of disease relapse [1, 2]. For children who tolerate $75 \mathrm{mg} / \mathrm{m}^{2} \mathrm{MP}$ without cytopenias the current UK trial for ALL (UKALL XI) includes a cycle of MP dose escalation, whereas the previous trial (UKALL X) did not [3]. Such dose escalation has not led to an increase in the cumulative dose of MP prescribed because it generates more gaps in treatment due to more frequent drug withdrawal [4]. We were concerned that the gaps in therapy produced by escalation might paradoxically reduce the cytotoxicity of MP, so were interested to see what effect escalation had on TGN concentrations. To find out we compared two consecutive cohorts of children who were treated according to UKALL X and UKALL XI respectively.

\section{Methods}

Consecutive eligible children with ALL who received continuing chemotherapy according to UKALL XI, and who could tolerate $75 \mathrm{mg} / \mathrm{m}^{2} \mathrm{MP}$ for over 4 weeks, were studied. We compared them with group of UKALL X children matched for order of diagnosis, MP tolerance and gender. Sex-matching was considered important because on similar prescribing criteria boys tolerate more MP than girls [4].

Daily oral MP therapy was similar in both trials with a standard dose of $75 \mathrm{mg} / \mathrm{m}^{2}(100 \% \mathrm{MP})$. The children underwent protocol directed dose attenuation in 
response to neutropenia and/or thrombocytopenia. The UKALL XI children differed in that if $100 \%$ MP was tolerated for 4 consecutive weeks it was increased to $125 \%$ standard dose, and then monthly in further increments of $25 \%$ until cytopenia was induced.

Blood samples for the measurement of red blood cell (RBC) TGNs were obtained after at least 2 months of MP therapy when MP had been prescribed at the standard $100 \%$ dose or higher for at least the preceding 7 days, and when at least 2 months had elapsed since a RBC transfusion or a 5-day 'block' of intensive therapy [3]. All patient procedures were approved by the local Ethics Committee.

RBC TGNs were measured as previously described [5]. The lower limit of reproducibility was $30 \mathrm{pmol} /$ $8 \times 10^{8} \mathrm{RBCs}$ (interassay coefficient of variation $5.8 \%$ ). Medians were compared by the Mann-Whitney test.

\section{Results}

Four children (two from each trial) were not eligible for the study. They could not tolerate $100 \% \times 4$ weeks MP. TGN concentrations at their maximum MP dosage ranged from 500 to $1140 \mathrm{pmol} / 8 \times 10^{8} \mathrm{RBCs}$. Twentyone UKALL XI children ( 7 girls and 14 boys) were studied. From them 139 TGN assays were obtained at $100 \%$ MP (median 6 per child) and 104 assays at 125\% (median 5 per child). TGNs were compared in the first 100 to $125 \%$ escalation cycle (one reading per child). The TGN range at $100 \%$ was $113-520$, and at $125 \%$ $135-637 \mathrm{pmol} / 8 \times 10^{8} \mathrm{RBCs}$ with a median difference of 90 pmol $(95 \% \mathrm{CI}=25$ to $165 \mathrm{pmol}, P<0.02)$.

Eleven children required titration to $150 \%$ at some point in the study, three to $175 \%$ and one to $200 \%$. The three children titrated beyond 150\% MP had lower TGN concentrations at higher doses than at $100 \%$ dose. One child had TGN concentrations at 200\% dosages half those recorded at $125 \%$ MP. Partial compliance was suspected, and admitted in one case.

The UKALL XI children were then compared with a group of 21 UKALL X children who tolerated 100\% MP for 4 weeks or more. The UKALL X children had a total of 99 assays (median 5 per child) at 100\% MP. The UKALL XI children spent a median of $37 \%$ of their time on cytopenia induced MP dose reduction or withdrawal whereas the figure for UKALL X children was $27 \%$ (median difference $7.5 \%, 95 \% \mathrm{CI}-1.4$ to $14 \%, P=0.09)$. Cytopenia-driven dose reductions were more frequent in the UKALL XI children, but this did not reach significance [4].

There was no difference in TGN concentrations between UKALL XI or X taking either a single assay per child (the first), or the median value of all $100 \%$ assays for each child. There was a difference if just the TGN assays taken at the time cytopenia occurred were considered. For the UKALL X children 56 such assays were available (median 3 per child) and for the UKALL XI children 74 (median 4 per child). Comparison of the median values gave a difference of 78 pmol $(95 \%$ CI 20 to 144 pmol, $P<0.02)$ (Table 1$)$. The UKALL XI children had accumulated significantly higher concentrations of TGNs at the stage when their blood counts dropped.

\section{Discussion}

Continuing 'maintenance' chemotherapy is an important component of successfull ALL protocols $[1,6]$ and MP dose escalation is an attempt to avoid inadvertent under-treatment. Our findings provide some reassurance that, even if it produces more gaps in an ideally continuous schedule [4], escalation produces greater peaks in intracellular TGN concentrations which could translate into greater efficacy due to the long half life of such metabolites [1]. Whether that hope is realised remains to be seen.

It is worrying that three children on escalated MP doses $(>150 \%)$ had lower metabolite assay values at higher MP doses than at lower dosages and we regard this as evidence of partial compliance. This was admitted by one child. There are many other possible reasons for variation in intracellular metabolites, and we acknowledge that our suspicions are based on circumstantial evidence. Recognition of non-compliance is very important because if non-compliant children suddenly start to take an inflated dose they could suffer profound myelosuppressive toxicity.

This work was supported by the Leukaemia Research Fund of Great Britain.

Table 1 A comparison of TGN concentrations (range (median)) in UKALL X and XI

$T G N\left(p m o l / 8 \times 10^{8} \mathrm{RBCs}\right)$

\begin{tabular}{|c|c|c|c|}
\hline & \multicolumn{3}{|c|}{$T G N\left(\mathrm{pmol} / 8 \times 10^{8} \mathrm{RBCs}\right)$} \\
\hline & First assay at $100^{\circ} \% \mathrm{MP}$ & Median of all $100 \%$ assays & $\begin{array}{l}\text { Median of all assays at } \\
\text { time of dose reduction }\end{array}$ \\
\hline UKALL X & 156 to $629(323)$ & 156 to $545(317)$ & 246 to $503(317)$ \\
\hline UKALL XI & 113 to $520(317)$ & 116 to $536(293)$ & 270 to $643(385)$ \\
\hline Median difference & 24 & 26 & $78(95 \% \mathrm{CI}=20$ to 144$)$ \\
\hline Two-tail $P=$ & 0.46 & 0.41 & 0.017 \\
\hline
\end{tabular}




\section{References}

1 Lilleyman JS, Lennard L. Mercaptopurine metabolism and risk of relapse in childhood lymphoblastic leukaemia. Lancet 1994; 343: 1188-1190.

2 Lennard L, Lilleyman JS, Van Loon J, Weinshilboum RM. Genetic variation in response to 6-mercaptopurine for childhood acute lymphoblastic leukaemia. Lancet 1990; 336: $225-229$.

3 Chessells JM, Bailey CC, Richards SM. Intensification of treatment improves survival for all children with lymphoblastic leukaemia: results of MRC UKALL X. Lancet 1995; 345: $143-148$.

4 Welch JC, Lilleyman JS. 6-Mercaptopurine dose escalation and its effect on drug tolerance in childhood lymphoblastic leukaemia. Cancer Chemother Pharmacol 1996; 38: 113-116. 5 Lennard L, Singleton HJ. High-performance liquid chromatographic assay of the methyl and nucleotide metabolites of 6-mercaptopurine: quantification of red blood cell 6-thioguanine nucleotide, 6-thioinosinic acid and 6-methylmercaptopurine metabolites in a single sample. J Chromatogr 1992; 583: 83-90.

6 Gale RP, Butturini A. Maintenance chemotherapy and cure of childhood acute lymphoblastic leukaemia. Lancet 1991; 383: $1315-1318$.

(Received 11 January 1996, accepted 28 May 1996) 